\title{
Orbital and atmospheric characterization of the planet within the gap of the PDS 70 transition disk ${ }^{\star}$
}

\author{
A. Müller ${ }^{1}$, M. Keppler ${ }^{1}$, Th. Henning ${ }^{1}$, M. Samland ${ }^{1}$, G. Chauvin ${ }^{2,3}$, H. Beust ${ }^{2}$, A.-L. Maire ${ }^{1}$, K. Molaverdikhani ${ }^{1}$, \\ R. van Boekel ${ }^{1}$, M. Benisty 2,3 , A. Boccaletti ${ }^{4}$, M. Bonnefoy ${ }^{2}$, F. Cantalloube ${ }^{1}$, B. Charnay ${ }^{4}$, J.-L. Baudino ${ }^{5}$,

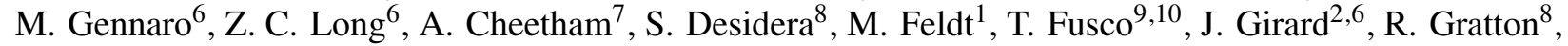 \\ J. Hagelberg ${ }^{11}$, M. Janson ${ }^{1,12}$, A.-M. Lagrange ${ }^{2}$, M. Langlois ${ }^{13,14}$, C. Lazzoni ${ }^{8}$, R. Ligi ${ }^{15}$, F. Ménard ${ }^{2}$, D. Mesa ${ }^{8,16}$, \\ M. Meyer ${ }^{11,17}$, P. Mollière ${ }^{18}$, C. Mordasini ${ }^{19}$, T. Moulin ${ }^{2}$, A. Pavlov ${ }^{1}$, N. Pawellek ${ }^{1,20}$, S. P. Quanz ${ }^{11}$, J. Ramos ${ }^{1}$, \\ D. Rouan ${ }^{4}$, E. Sissa ${ }^{8}$, E. Stadler ${ }^{2}$, A. Vigan ${ }^{21}$, Z. Wahhaj ${ }^{22}$, L. Weber ${ }^{7}$, and A. Zurlo ${ }^{23,24,10}$
}

(Affiliations can be found after the references)

Received 06 June 2018 / Accepted 08 July 2018

\begin{abstract}
Context. The observation of planets in their formation stage is a crucial but very challenging step in understanding when, how, and where planets form. PDS 70 is a young pre-main sequence star surrounded by a transition disk, in the gap of which a planetary-mass companion has recently been discovered. This discovery represents the first robust direct detection of such a young planet, possibly still at the stage of formation.

Aims. We aim to characterize the orbital and atmospheric properties of PDS $70 \mathrm{~b}$, which was first identified on May 2015 in the course of the SHINE survey with SPHERE, the extreme adaptive-optics instrument at the VLT.

Methods. We obtained new deep SPHERE/IRDIS imaging and SPHERE/IFS spectroscopic observations of PDS $70 \mathrm{~b}$. The astrometric baseline now covers $6 \mathrm{yr}$, which allowed us to perform an orbital analysis. For the first time, we present spectrophotometry of the young planet which covers almost the entire near-infrared range $(0.96-3.8 \mu \mathrm{m})$. We use different atmospheric models covering a large parameter space in temperature, log $g$, chemical composition, and cloud properties to characterize the properties of the atmosphere of PDS $70 \mathrm{~b}$.

Results. PDS $70 \mathrm{~b}$ is most likely orbiting the star on a circular and disk coplanar orbit at $\sim 22$ au inside the gap of the disk. We find a range of models that can describe the spectrophotometric data reasonably well in the temperature range $1000-1600 \mathrm{~K}$ and $\log g$ no larger than 3.5 dex. The planet radius covers a relatively large range between 1.4 and $3.7 R_{\mathrm{J}}$ with the larger radii being higher than expected from planet evolution models for the age of the planet of $5.4 \mathrm{Myr}$.

Conclusions. This study provides a comprehensive data set on the orbital motion of PDS $70 \mathrm{~b}$, indicating a circular orbit and a motion coplanar with the disk. The first detailed spectral energy distribution of PDS $70 \mathrm{~b}$ indicates a temperature typical of young giant planets. The detailed atmospheric analysis indicates that a circumplanetary disk may contribute to the total planetflux.
\end{abstract}

Key words. planets and satellites: atmospheres - planets and satellites: individual: PDS 70 - techniques: spectroscopic - astrometry - methods: observational

\section{Introduction}

Our knowledge of the formation mechanism and evolution of planets has developed by leaps and bounds since the first detection of an exoplanet by Mayor \& Queloz (1995) around the main-sequence star 51 Peg. However, constraining the formation timescales, the location of planet formation, and the physical properties of such objects remains a challenge and to date has mostly been based on indirect arguments using measured properties of protoplanetary disks. What is really needed is a detection of planets around young stars still surrounded by a disk. Modern coronagraphic angular differential imaging surveys that utilize extreme adaptive optics, such as the SpHere INfrared survey for Exoplanets (SHINE; Chauvin et al. 2017), provide the necessary spatial resolution and sensitivity to find such young planetary systems.

In Keppler et al. (2018) we reported the first bona fide detection of a giant planet inside the gap of the transition disk

\footnotetext{
* Based on observations collected at the European Organisation for Astronomical Research in the Southern Hemisphere under ESO programmes 095.C-0298, 097.C-0206, 097.C-1001, 1100.C-0481.
}

around the star PDS 70 together with the characterization of its protoplanetary disk. PDS 70 is a K7-type $5.4 \mathrm{Myr}$ young premain sequence member of the Upper Centaurus-Lupus group (Riaud et al. 2006; Pecaut \& Mamajek 2016) at a distance of distance of $113.43 \pm 0.52 \mathrm{pc}$ (Gaia Collaboration 2016, 2018). Our determination of the stellar parameters are explained in detail in Appendix A. The planet was detected in five epochs with VLT/SPHERE (Beuzit et al. 2008), VLT/NaCo (Lenzen et al. 2003; Rousset et al. 2003), and Gemini/NICI (Chun et al. 2008) covering a wavelength range from $H$ to $L^{\prime}$ band. In this paper we present new deep $K$-band imaging and first $Y-H$ spectroscopic data with SPHERE with the goal of putting constraints on the orbital parameters and properties of PDS $70 \mathrm{~b}$.

\section{Observations and data reduction}

\subsection{Observations}

We observed PDS 70 during the SPHERE/SHINE GTO program on the night of February 24, 2018. The data were taken in the IRDIFS-EXT pupil tracking mode using the 
N_ALC_YJH_S (185 mas in diameter) apodized-Lyot coronagraph (Martinez et al. 2009; Carbillet et al. 2011). We used the IRDIS (Dohlen et al. 2008) dual-band imaging camera (Vigan et al. 2010) with the $K_{1} K_{2}$ narrow-band filter pair $\left(\lambda_{K_{1}}=2.110 \pm 0.102 \mu \mathrm{m}, \lambda_{K_{2}}=2.251 \pm 0.109 \mu \mathrm{m}\right)$. A spectrum covering the spectral range from $Y$ to $H$ band $(0.96-1.64 \mu \mathrm{m}$, $R_{\lambda}=30$ ) was acquired simultaneously with the IFS integral field spectrograph (Claudi et al. 2008). We set the integration time for both detectors to $96 \mathrm{~s}$ and acquired a total time on target of almost $2.5 \mathrm{~h}$. The total field rotation is $95.7^{\circ}$. During the course of observation the average coherence time was $7.7 \mathrm{~ms}$ and a Strehl ratio of $73 \%$ was measured at $1.6 \mu \mathrm{m}$, providing excellent observing conditions.

\subsection{Data reduction}

The IRDIS data were reduced as described in Keppler et al. (2018). The basic reduction steps consisted of bad-pixel correction, flat fielding, sky subtraction, distortion correction (Maire et al. 2016), and frame registration.

The IFS data were reduced with the SPHERE Data Center pipeline (Delorme et al. 2017), which uses the Data Reduction and Handling software (v0.15.0, Pavlov et al. 2008) and additional IDL routines for the IFS data reduction (Mesa et al. 2015). The modeling and subtraction of the stellar speckle pattern for both the IRDIS and IFS data set were performed with a smart Principal Component Analysis (sPCA) algorithm based on Absil et al. (2013) using the same setup as described in Keppler et al. (2018). Figure 1 shows the high-quality IRDIS combined $K_{1} K_{2}$ image of PDS 70. The outer disk and the planetary companion inside the gap are clearly visible. In addition, there are several disk related features present, which are described in Appendix B. For this image the data were processed with a classical ADI reduction technique (Marois et al. 2006) to minimize self-subtraction of the disk. The extraction of astrometric and contrast values was performed by injecting negative point source signals into the raw data (using the unsaturated flux measurements of PDS 70) which were varied in contrast and position based on a predefined grid created from a first initial estimate of the planet's contrast and position. For every parameter combination of the inserted negative planet the data were reduced with the same sPCA setup (maximum of 20 modes, protection angle of $0.75 \times F W H M$ ) and a $\chi^{2}$ value within a segment of $2 \times F W H M$ and $4 \times F W H M$ around the planet's position was computed. Following Olofsson et al. (2016), the marginalized posterior probability distributions for each parameter was computed to derive final contrast and astrometric values and their corresponding uncertainties (the uncertainties correspond to the $68 \%$ confidence interval). For an independent confirmation of the extracted astrometry and photometry we used SpeCal (Galicher et al. 2018) and find the values in good agreement with each other within $1 \sigma$ uncertainty.

\subsection{Conversion of the planet contrasts to physical fluxes}

The measured contrasts of PDS $70 \mathrm{~b}$ from all data sets (SPHERE, $\mathrm{NaCo}$, and NICI) were converted to physical fluxes following the approach used in Vigan et al. (2016) and Samland et al. (2017), who used a synthetic spectrum calibrated by the stellar SED to convert the measured planet contrasts at specific wavelengths to physical fluxes. In our case, instead of a synthetic spectrum, which does not account for any (near-)infrared excess, we made use of the flux calibrated spectrum of PDS 70 from the SpeX spectrograph (Rayner et al. 2003), which is presented in

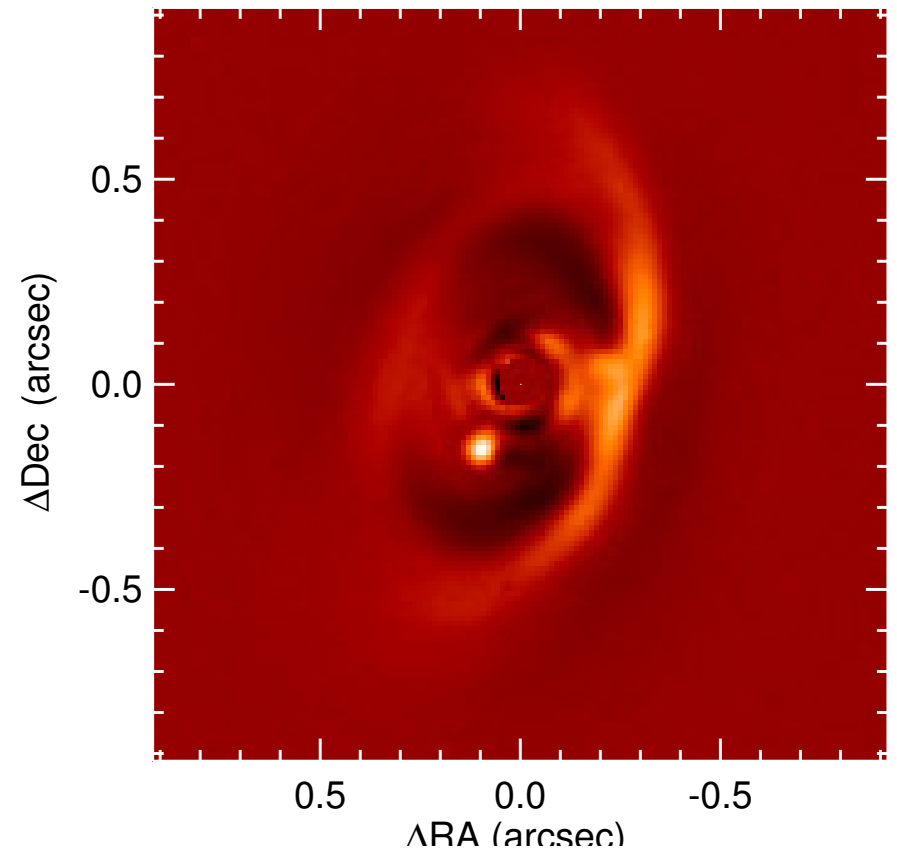

Fig. 1. IRDIS combined $K_{1} K_{2}$ image of PDS 70 using classical ADI reduction technique showing the planet inside the gap of the disk around PDS 70. The central part of the image is masked out for better display. North is up, East is to the left.

Long et al. (2018). The spectrum Long et al. (2018). The spectrum covers a wavelength the entire IFS and IRDIS data set. To obtain flux values for our data sets taken in $L^{\prime}$ band at $3.8 \mu \mathrm{m}$, we modeled the stellar SED with simple blackbodies to account for the observed infrared excess (Hashimoto et al. 2012; Dong et al. 2012). The final SED of the planet is shown in Fig. 2. The IFS SED of the planet is shown in Fig. 2. The IFS spectrum has a steep slope and displays a few features only, mainly water values are listed in Table C.1.

\section{Results}

\subsection{Atmospheric modeling}

We performed atmospheric simulations for PDS $70 \mathrm{~b}$ with the selfconsistent $1 \mathrm{D}$ radiative-convective equilibrium tool petitCODE (Mollière et al. 2015, 2017), which resulted in three different grids of self-luminous cloudy planetary model atmospheres (see Table 1). These grids mainly differ in the treatment of clouds: petitCODE(1) does not consider scattering and includes only $\mathrm{Mg}_{2} \mathrm{SiO}_{4}$ cloud opacities; petitCODE(2) adds scattering; petit$C O D E(3)$ contains four more cloud species including iron $\left(\mathrm{Na}_{2} \mathrm{~S}\right.$, $\left.\mathrm{KCl}, \mathrm{Mg}_{2} \mathrm{SiO}_{4}, \mathrm{Fe}\right)$. Additionally, we also use the publicly available cloud-free petitCODE model grid (here called petitCODE $(0)$; see Samland et al. 2017 for a detailed description of this grid) and the public PHOENIX BT-Settl grid (Allard 2014; Baraffe et al. 2015).

In order to compare the data to the petitCODE models we use the same tools as described in Samland et al. (2017), using the python MCMC code emcee (Foreman-Mackey et al. 2013) on $N$-dimensional model grids linearly interpolated at each evaluation. We assume a Gaussian likelihood function and take into account the spectral correlation of the IFS spectra (Greco \& Brandt 2016). For an additional independent confirmation of the results obtained using petitCODE, we also used cloudy models from the Exo-REM code. The models 


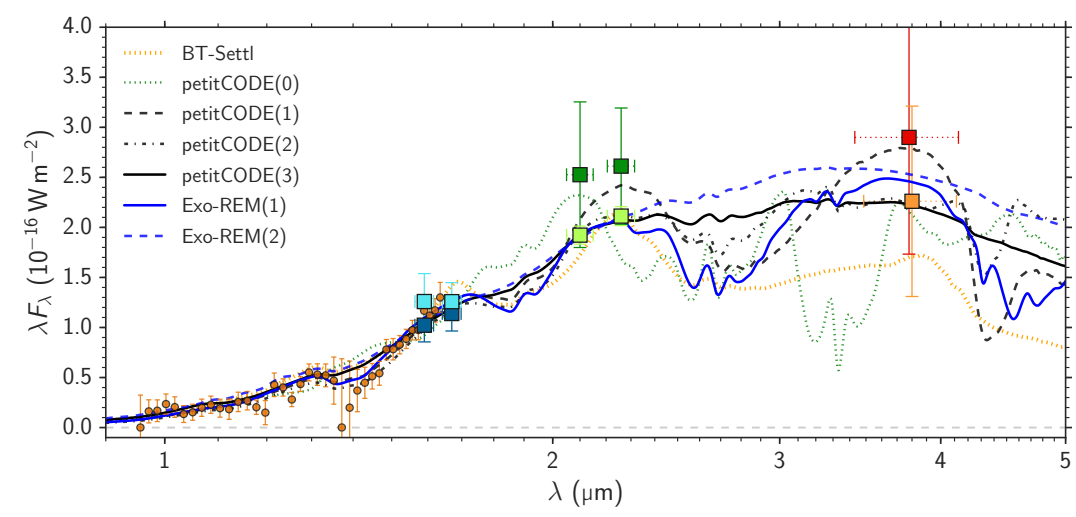

Fig. 2. Spectral energy distribution of PDS $70 \mathrm{~b}$ as a function of wavelength constructed from $Y$ - to $H$-band IFS spectra (orange points), IRDIS $\mathrm{H}_{2} \mathrm{H}_{3}$ (first epoch in dark blue, second epoch in light blue), and $K_{1} K_{2}$ (first epoch in dark green, second epoch in light green), $\mathrm{NaCo}$ (red), and NICI (orange) $L^{\prime}$-band images. Plotted are the best fits for the seven model grids smoothed to the resolution of IFS.

Table 1. Model grids used as input for MCMC exploration.

\begin{tabular}{|c|c|c|c|c|c|c|c|c|c|}
\hline Model & $\begin{array}{l}T_{\text {eff }} \\
(\mathrm{K}) \\
\end{array}$ & $\begin{array}{l}\Delta T \\
(\mathrm{~K}) \\
\end{array}$ & $\begin{array}{c}\log g \\
\log _{10}(\mathrm{cgs})\end{array}$ & $\begin{array}{c}\Delta \log g \\
\log _{10}(\mathrm{cgs})\end{array}$ & $\begin{array}{c}(M / H) \\
(\operatorname{dex})\end{array}$ & $\begin{array}{c}\Delta(M / H) \\
(\operatorname{dex})\end{array}$ & $f_{\text {sed }}$ & $\Delta f_{\text {sed }}$ & Remarks \\
\hline BT-Settl & $1200-3000$ & 100 & $3.0-5.5$ & 0.5 & 0.0 & - & - & - & - \\
\hline petitCODE $(0)$ & $500-1700$ & 50 & $3.0-6.0$ & 0.5 & -1.0 to 1.4 & 0.2 & - & - & Cloud-free \\
\hline petitCODE (1) & $1000-1500$ & 100 & $2.0-5.0$ & 1.0 & -1.0 to 1.0 & 1.0 & 1.5 & - & w/o scattering, w/o Fe clouds \\
\hline petitCODE(2) & $1000-1500$ & 100 & $2.0-5.0$ & 0.5 & $0.0-1.5$ & 0.5 & $0.5-6.0$ & $1.0^{a}$ & with scattering, w/o Fe clouds \\
\hline petitCODE(3) & $1000-2000$ & 200 & $3.5-5.0$ & 0.5 & -0.3 to 0.3 & 0.3 & 1.5 & - & With scattering, with Fe clouds \\
\hline Exo-REM(1) & $400-2000$ & 100 & $3.0-5.0$ & 0.1 & $0.32,1.0,3.32$ & - & - & - & Cloud particle size fixed to $20 \mu \mathrm{m}$ \\
\hline $\operatorname{Exo-REM}(2)$ & $400-2000$ & 100 & $3.0-5.0$ & 0.1 & $0.32,1.0,3.32$ & - & 1.0 & - & - \\
\hline
\end{tabular}

Notes. The radius of the planet was included as an additional analytic fit-parameter regardless of the model, ranging from $0.1 R_{\mathrm{J}}$ to $5 R_{\mathrm{J}}{ }^{(a)}$ Except additional grid point at 0.5 .

Table 2. Parameters of best-fit models based on the grids listed in Table 1.

\begin{tabular}{lcccccccc}
\hline \hline Model & $\begin{array}{c}T_{\text {eff }} \\
(\mathrm{K})\end{array}$ & $\begin{array}{c}\log g \\
\log _{10}(\mathrm{cgs})\end{array}$ & $\begin{array}{c}(M / H) \\
(\mathrm{dex})\end{array}$ & $f_{\text {sed }}$ & $\begin{array}{c}\text { Radius } \\
R_{\mathrm{J}}\end{array}$ & $\begin{array}{c}\text { Mass }^{b} \\
M_{\mathrm{J}}\end{array}$ & $K$ flux & $L^{\prime}$ flux \\
\hline BT-Settl & 1590 & 3.5 & - & - & 1.4 & 2.4 & Yes & Yes \\
petitCODE(0) & 1155 & 5.5 & -1.0 & - & 2.7 & 890.0 & No & $($ Yes $)$ \\
petitCODE $(1)$ & 1050 & $\leq 2.0$ & $\geq 1.0$ & $1.5^{a}$ & 2.0 & 0.2 & Yes & Yes \\
petitCODE(2) & 1100 & 2.65 & 1.0 & 1.24 & 3.3 & 1.9 & Yes & $($ No) \\
petitCODE(3) & 1190 & $\leq 3.5$ & 0.0 & $\leq 1.5$ & 2.7 & 8.9 & Yes & Yes \\
Exo-REM(1) & 1000 & 3.5 & 1.0 & - & 3.7 & 17 & Yes & Yes \\
Exo-REM(2) & 1100 & 4.1 & 1.0 & 1 & 3.3 & 55 & Yes & Yes \\
\hline
\end{tabular}

Notes. The last two columns indicate qualitatively whether the corresponding model is compatible with the photometric points in $K$ and $L^{\prime}$ band, whereas all models describe the $Y$ - to $H$-band data well. ${ }^{(a)}$ Only grid value. ${ }^{(b)}$ As derived from $\log g$ and radius

and corresponding simulations are described in Charnay et al. (2018). Exo-REM assumes non-equilibrium chemistry, and silicate and iron clouds. For the model grid Exo-REM(1) the cloud particles are fixed at $20 \mu \mathrm{m}$ and the vertical distribution takes into account vertical mixing (with a parametrized $K_{z z}$ ) and sedimentation. The Exo-REM(2) model uses a cloud distribution with a fixed sedimentation parameter $f_{\text {sed }}=1$ as in the model by Ackerman \& Marley (2001) and petitCODE. Table 2 provides a compilation of the best-fit values and Fig. 2 shows the respective spectra. The values quoted correspond to the peak of the respective marginalized posterior probability distribution. The cloud-free models fail to represent the data and result in unphysical parameters. In contrast, the cloudy models provide a much better representation of the data. The results obtained by the petitCODE and Exo-REM models are consistent with each other. However, because of higher cloud opacities in the Exo-REM(2) models the $\log g$ values are less constrained and the water feature at $1.4 \mu \mathrm{m}$ is less pronounced. Therefore, the resulting spectrum is closer to a blackbody and the resulting mass is less constrained. All these models indicate a relatively low temperature and surface gravity, but in some cases unrealistically high radii. Evolutionary models predict radii smaller than $2 R_{\mathrm{J}}$ for planetary-mass objects (Mordasini et al. 2017). The radius can be pushed toward lower values if cloud opacities are removed, for example by removing iron (petitCODE(2)). However, a direct comparison for the same model parameters shows that this effect is very small. In petitCODE(1) this is shown in an exaggerated way by artificially removing scattering from the models, which leads to a significant reduction in radius. In general, we find a wide range of models that are compatible with the current data. The parts of the spectrum most suitable for ruling out models are the possible water absorption feature at $1.4 \mu \mathrm{m}$, and the spectral behavior at longer wavelengths $(K$ to $L^{\prime}$ band). Given the low signal-to-noise ratio in the water 


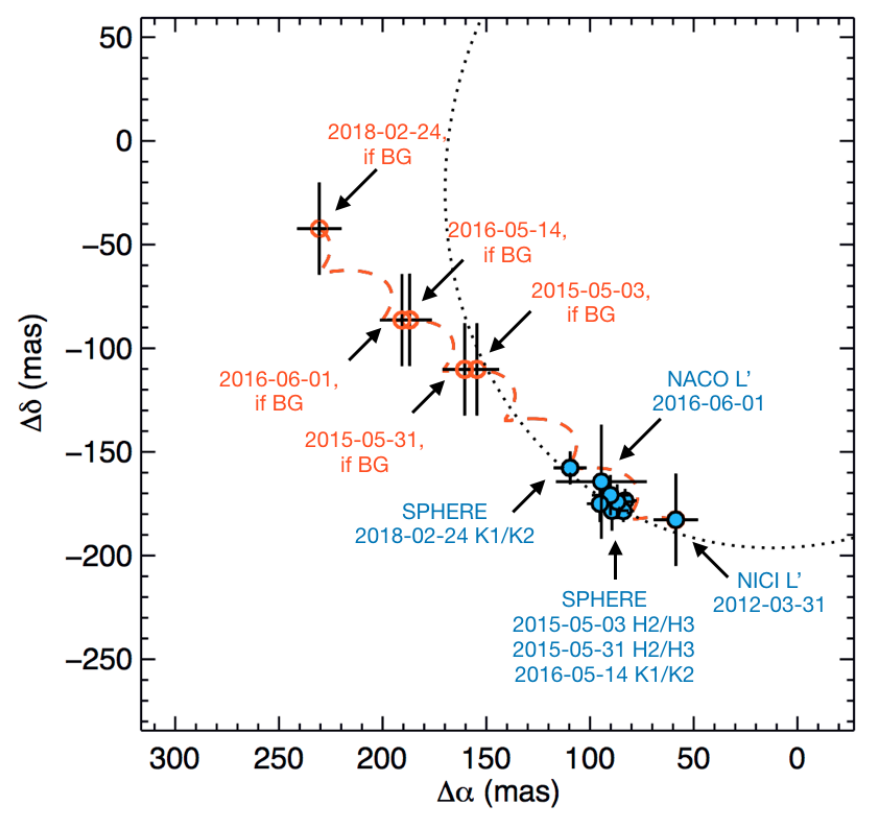

Fig. 3. Multi-epoch astrometric measurements of PDS $70 \mathrm{~b}$ relative to PDS 70 (in blue). The plot also shows the predictions of the relative position under the hypothesis of a stationary background star for the same observing dates (in red). The gray dotted line shows one of the most likely orbital solutions based on our MCMC analysis (see text for details).

absorption feature and the large uncertainties in the $L^{\prime}$ flux, it is very challenging to draw detailed physical conclusions about the nature of the object. We emphasize that other possible explanations for the larger than expected radii from evolutionary models include the recent accretion of material, additional reddening by circumplanetary material, and significant flux contributions from a potential circumplanetary disk. The third possibility is especially interesting in the light of possible features in our reduced images that could present spiral arm structures close to the planet (Fig. 1). There also appears to be an increase in $\mathrm{HCO}^{+}$velocity dispersion close to the location of the planet in the ALMA data presented by Long et al. (2018).

\subsection{Orbital properties of PDS $70 \mathrm{~b}$}

The detailed results of the relative astrometry and photometry extracted from our observation from February 2018 are listed in Table C.1 together with the earlier epochs presented in Keppler et al. (2018). A first verification of the relative position of PDS $70 \mathrm{~b}$ with what we could expect for a stationary background contaminant is shown in Fig. 3. The latest SPHERE observations of February 24, 2018, confirms that the companion is comoving with the central star.

To explore the possible orbital solutions of PDS $70 \mathrm{~b}$, we applied the Markov chain Monte Carlo (MCMC) Bayesian analysis technique (Ford 2005, 2006) developed for $\beta$ Pictoris (Chauvin et al. 2012), which is well suited for observations covering a small part of the whole orbit (for large orbital periods), as in the case of PDS 70b. We did not initially consider any prior information on the inclination or longitude of ascending node to explore the full orbital parameter space of bound orbits. As described in Appendix A of Chauvin et al. (2012), we assume the prior distribution $p_{0}(x)$ to be uniform in $x=\left(\log P, e, \cos i, \Omega+\omega, \omega-\Omega, t_{\mathrm{p}}\right)$ and work on a modi- fied parameter vector $\boldsymbol{u}(\boldsymbol{x})$ to avoid singularities in inclination and eccentricities and to improve the convergence of the Markov chains. The results of the MCMC analysis are shown in Fig. D.1, together with the results of a classical least-squares linear method (LSLM) flagged by the red line. It shows the standard statistical distribution matrix of the orbital elements $a, e$, $i, \Omega, \omega$, and $t_{\mathrm{p}}$, where $a$ stands for the semi-major axis, $e$ for the eccentricity, $i$ for the inclination, $\Omega$ the longitude of the ascending node (measured from north), $\omega$ the argument of periastron, and $t_{\mathrm{p}}$ the time for periastron passage. The results of our MCMC fit (Table D.1) indicate orbital distributions that peak at $22.2_{-9.7}^{+6.2}$ au (the uncertainties correspond to the $68 \%$ confidence interval) for the semi-major axis, $151.1_{-13.6}^{+14.1 \circ}$ for the inclination, and eccentricities compatible with low-eccentric solutions as shown by the $(a, e)$ correlation diagram. The elements $\Omega$ and $\omega$ are poorly constrained as low-eccentric solutions are favored and as pole-on solutions are also likely possible. Time at periastron is poorly constrained. The inclination distribution clearly favors retrograde orbits $\left(i>90^{\circ}\right)$, which is compatible with the observed clockwise orbital motion resolved with SPHERE, NaCo, and NICI. To consider the disk geometry described by Keppler et al. (2018), we decided to explore the MCMC solutions compatible with a planet-disk coplanar configuration. We restrained the PDS $70 \mathrm{~b}$ solution set given by the MCMC to those solutions with orbital plane making a tilt angle less than $5^{\circ}$ with respect to the disk midplane described by Keppler et al. (2018), i.e., $i=180^{\circ}-49.8^{\circ}$ and $\mathrm{PA}=158.6^{\circ}$. The results are shown in Fig. D. 2 and Table D. 1 together with the relative astrometry of PDS $70 \mathrm{~b}$ reported with 200 orbital solutions randomly drawn from our MCMC distributions in Fig. D.4. Figure D.3 shows the posterior distribution (out of Fig. D.1) of the tilt angle with the disk plane assuming $i_{\text {disk }}=130.2^{\circ}$ and $\mathrm{PA}=158.6^{\circ}$. The distribution peaks around $50^{\circ}$, which remains consistent with a likely coplanar planet-disk configuration (or a moderate tilt angle) given the uncertainties. Given the small fraction of orbit covered by our observations, a broad range of orbital configurations are possible including coplanar solutions that could explain the formation of the broad disk cavity carved by PDS $70 \mathrm{~b}$.

\section{Summary and conclusions}

We presented new deep SPHERE/IRDIS imaging data and, for the first time, SPHERE/IFS spectroscopy of the planetary mass companion orbiting inside the gap of the transition disk around PDS 70. With the accurate distance provided by Gaia DR2 we derived new estimates for the stellar mass $\left(0.76 \pm 0.02 M_{\odot}\right)$ and age $(5.4 \pm 1.0 \mathrm{Myr})$. Taking into account the data sets presented in Keppler et al. (2018) we achieve an orbital coverage of $6 \mathrm{yr}$. Our MCMC Bayesian analysis favors a circular $\sim 22$ au wide and a disk coplanar orbit, which translates to an orbital period of $118 \mathrm{yr}$. The new imaging data show rich details in the structure of the circumstellar disk. Several arcs and potential spirals can be identified (see Fig. B.1). Determining the way these features are connected to the presence of the planet is beyond the scope of this study. With the new IFS spectroscopic data and photometric measurements from previous IRDIS, NaCo, and NICI observations we were able to construct a SED of the planet covering a wavelength range of $0.96-3.8 \mu \mathrm{m}$. We computed three sets of cloudy model grids with the petitCODE and two models with Exo-REM with different treatment of clouds. These model grids and the BT-Settl grid were fitted to the planet's SED. The atmospheric analysis clearly demonstrates that cloud-free models do not provide a good fit to the data. In contrast, we find a range 
of cloudy models that can describe the spectrophotometric data reasonably well, and result in a temperature range of 1000$1600 \mathrm{~K}$ and $\log g$ no larger than $3.5 \mathrm{dex}$. The radius varies significantly between 1.4 and $3.7 R_{\mathrm{J}}$ based on the model assumptions and is in some cases higher than what we expect from evolutionary models. The planet's mass derived from the best-fit values ranges from 2 to $17 M_{\mathrm{J}}$, which is similar to the masses derived from evolutionary models by Keppler et al. (2018). This paper provides the first step toward a comprehensive characterization of the orbit and atmospheric parameters of an embedded young planet. Observations with JWST and ALMA will provide additional constraints on the nature of this object, especially in the presence of a circumplanetary disk.

Acknowledgements. SPHERE is an instrument designed and built by a consortium consisting of IPAG (Grenoble, France), MPIA (Heidelberg, Germany), LAM (Marseille, France), LESIA (Paris, France), Laboratoire Lagrange (Nice, France), INAF-Osservatorio di Padova (Italy), Observatoire de Genève (Switzerland), ETH Zurich (Switzerland), NOVA (Netherlands), ONERA (France) and ASTRON (Netherlands) in collaboration with ESO. SPHERE was funded by ESO, with additional contributions from CNRS (France), MPIA (Germany) INAF (Italy), FINES (Switzerland) and NOVA (Netherlands). SPHERE also received funding from the European Commission Sixth and Seventh Framework Programmes as part of the Optical Infrared Coordination Network for Astronomy (OPTICON) under grant number RII3-Ct-2004-001566 for FP6 (2004 2008), grant number 226604 for FP7 (2009-2012) and grant number 312430 for FP7 (2013-2016). We also acknowledge financial support from the Programme National de Planétologie (PNP) and the Programme National de Physique Stellaire (PNPS) of CNRS-INSU in France. This work has also been supported by a grant from the French Labex OSUG@2020 (Investissements d'avenir-ANR10 LABX56). The project is supported by CNRS, by the Agence Nationale de la Recherche (ANR-14-CE33-0018). It has also been carried out within the frame of the National Centre for Competence in Research PlanetS supported by the Swiss National Science Foundation (SNSF). M.R.M., H.M.S., and S.D are pleased to acknowledge the financial support of the SNSF. Finally, this work has made use of the the SPHERE Data Centre, jointly operated by OSUG/IPAG (Grenoble), PYTHEAS/LAM/CESAM (Marseille), OCA/Lagrange (Nice), and Observtoire de Paris/LESIA (Paris). We thank P. Delorme and E. Lagadec (SPHERE Data Centre) for their efficient help during the data reduction process. This work has made use of the SPHERE Data Centre, jointly operated by OSUG/IPAG (Grenoble), PYTHEAS/LAM/CeSAM (Marseille), OCA/Lagrange (Nice) and Observatoire de Paris/LESIA (Paris) and supported by a grant from Labex OSUG@2020 (Investissements d'avenir-ANR10 LABX56). A.M acknowledges the support of the DFG priority program SPP 1992 "Exploring the Diversity of Extrasolar Planets" (MU 4172/1-1). F.Me. and M.B. acknowledge funding from ANR of France under contract number ANR-16-CE31-0013. D.M. acknowledges support from the ESO-Government of Chile Joint Comittee program "Direct imaging and characterization of exoplanets". J.L.B. acknowledges the support of the UK Science and Technology Facilities Council. A.Z. acknowledges support from the CONICYT+PAI/Convocatoria nacional subvención a la instalación en la academia, convocatoria 2017+Folio PAI77170087. We thank the anonymous referee for the constructive report on the manuscript. This work has made use of data from the European Space Agency (ESA) mission Gaia (https://www.cosmos.esa.int/gaia), processed by the Gaia Data Processing and Analysis Consortium (DPAC, https://www.cosmos.esa.int web/gaia/dpac/consortium). Funding for the DPAC has been provided by national institutions, in particular the institutions participating in the Gaia Multilateral Agreement. This research has made use of NASA's Astrophysics Data System Bibliographic Services of the SIMBAD database, operated at CDS, Strasbourg. Based on observations obtained at the Gemini Observatory (acquired through the Gemini Observatory Archive), which is operated by the Association of Universities for Research in Astronomy, Inc., under a cooperative agreement with the NSF on behalf of the Gemini partnership: the National Science Foundation (United States), the National Research Council (Canada), CONICYT (Chile), Ministerio de Ciencia, Tecnología e Innovación Productiva (Argentina), and Ministério da Ciência, Tecnologia e Inovação (Brazil).

\section{References}

Absil, O., Milli, J., Mawet, D., et al. 2013, A\&A, 559, L12 Ackerman, A. S., \& Marley, M. S. 2001, ApJ, 556, 872 Allard, F. 2014, IAU Symp., 299, 271
Baraffe, I., Homeier, D., Allard, F., \& Chabrier, G. 2015, A\&A, 577, A42

Beuzit, J.-L., Feldt, M., Dohlen, K., et al. 2008, Proc. SPIE, 7014, 701418 Carbillet, M., Bendjoya, P., Abe, L., et al. 2011, Exp. Astron., 30, 39

Cardelli, J. A., Clayton, G. C., \& Mathis, J. S. 1989, ApJ, 345, 245 Chabrier, G. 2003, PASP, 115, 763

Charnay, B., Bézard, B., Baudino, J.-L., et al. 2018, ApJ, 854, 172

Chauvin, G., Lagrange, A.-M., Beust, H., et al. 2012, A\&A, 542, A41

Chauvin, G., Desidera, S., Lagrange, A.-M., et al. 2017, in Proc. of the Annual Meeting of the SF2A-2017, eds. C. Reylé, P. Di Matteo, F. Herpin, et al., 331 Choi, J., Dotter, A., Conroy, C., et al. 2016, ApJ, 823, 102

Chun, M., Toomey, D., Wahhaj, Z., et al. 2008, Proc. SPIE, 7015, 70151V Claudi, R. U., Turatto, M., Gratton, R. G., et al. 2008, Proc. SPIE, 7014, 70143E Cutri, R. M., Skrutskie, M. F., van Dyk, S., et al. 2003, VizieR Online Data Catalog: II/246

Delorme, P., Meunier, N., Albert, D., et al. 2017, in Proc. of the Annual Meeting of the SF2A-2017, eds. C. Reylé, P. Di Matteo, F. Herpin, et al., 347

Dohlen, K., Langlois, M., Saisse, M., et al. 2008, Proc. SPIE, 7014, 70143L

Dong, R., Hashimoto, J., Rafikov, R., et al. 2012, ApJ, 760, 111

Dotter, A. 2016, ApJS, 222, 8

Ford, E. B. 2005, AJ, 129, 1706

Ford, E. B. 2006, ApJ, 642, 505

Foreman-Mackey, D., Hogg, D. W., Lang, D., \& Goodman, J. 2013, PASP, 125, 306

Gaia Collaboration (Prusti, T., et al.) 2016, A\&A, 595, A1

Gaia Collaboration (Brown, A. G. A., et al.) 2018, A\&A, 616, A1

Galicher, R., Boccaletti, A., Mesa, D., et al. 2018, A\&A, 615, A92

Greco, J. P., \& Brandt, T. D. 2016, ApJ, 833, 134

Hashimoto, J., Dong, R., Kudo, T., et al. 2012, ApJ, 758, L19

Henden, A. A., Levine, S., Terrell, D., \& Welch, D. L. 2015, in AAS Meeting, 225 , id.336.16

Keppler, M., Benisty, M., Müller, A., et al. 2018, A\&A, 617, A44

Lenzen, R., Hartung, M., Brandner, W., et al. 2003, Proc. SPIE, 4841, 944

Long, Z. C., Akiyama, E., Sitko, M., et al. 2018, ApJ, 858, 112

Maire, A.-L., Langlois, M., Dohlen, K., et al. 2016, Proc. SPIE, 9908, 990834

Marois, C., Lafrenière, D., Doyon, R., Macintosh, B., \& Nadeau, D. 2006, ApJ, 641, 556

Martinez, P., Dorrer, C., Aller Carpentier, E., et al. 2009, A\&A, 495, 363

Mayor, M., \& Queloz, D. 1995, Nature, 378, 355

Mesa, D., Gratton, R., Zurlo, A., et al. 2015, A\&A, 576, A121

Mollière, P., van Boekel, R., Dullemond, C., Henning, T., \& Mordasini, C. 2015, ApJ, 813, 47

Mollière, P., van Boekel, R., Bouwman, J., et al. 2017, A\&A, 600, A10

Mordasini, C., Marleau, G.-D., \& Mollière, P. 2017, A\&A, 608, A72

Olofsson, J., Samland, M., Avenhaus, H., et al. 2016, A\&A, 591, A108

Pavlov, A., Möller-Nilsson, O., Feldt, M., et al. 2008, Proc. SPIE, 7019, 701939

Paxton, B., Bildsten, L., Dotter, A., et al. 2011, ApJS, 192, 3

Paxton, B., Cantiello, M., Arras, P., et al. 2013, ApJS, 208, 4

Paxton, B., Marchant, P., Schwab, J., et al. 2015, ApJS, 220, 15

Pecaut, M. J., \& Mamajek, E. E. 2016, MNRAS, 461, 794

Rayner, J. T., Toomey, D. W., Onaka, P. M., et al. 2003, PASP, 115, 362

Riaud, P., Mawet, D., Absil, O., et al. 2006, A\&A, 458, 317

Rousset, G., Lacombe, F., Puget, P., et al. 2003, Proc. SPIE, 4839, 140

Samland, M., Mollière, P., Bonnefoy, M., et al. 2017, A\&A, 603, A57

Vigan, A., Moutou, C., Langlois, M., et al. 2010, MNRAS, 407, 71

Vigan, A., Bonnefoy, M., Ginski, C., et al. 2016, A\&A, 587, A55

1 Max Planck Institute for Astronomy, Königstuhl 17, 69117 Heidelberg, Germany

e-mail: amueller@mpia.de

2 Univ. Grenoble Alpes, CNRS, IPAG, 38000 Grenoble, France

3 Unidad Mixta Internacional Franco-Chilena de Astronomía, CNRS/INSU UMI 3386 and Departamento de Astronomía, Universidad de Chile, Casilla 36-D, Santiago, Chile

4 LESIA, Observatoire de Paris, Université PSL, CNRS, Sorbonne Université, Université Paris Diderot, Sorbonne Paris Cité, 5 place Jules Janssen, 9219 Meudon, France

5 Department of Physics, University of Oxford, Oxford, UK

6 Space Telescope Science Institute, 3700 San Martin Dr., Baltimore, MD 21218, USA

7 Geneva Observatory, University of Geneva, Chemin des Mailettes 51, 1290 Versoix, Switzerland 
8 INAF-Osservatorio Astronomico di Padova, Vicolo della Osservatorio 5, 35122 Padova, Italy

9 DOTA, ONERA, Université Paris Saclay, 91123 Palaiseau, France

10 Aix Marseille Université, CNRS, Laboratoire d'Astrophysique de Marseille (LAM) UMR 7326, 13388 Marseille, France

11 Institute for Particle Physics and Astrophysics, ETH Zurich, Wolfgang-Pauli-Strasse 27, 8093 Zurich, Switzerland

12 Department of Astronomy, Stockholm University, AlbaNova University Center, 10691 Stockholm, Sweden

13 Aix Marseille Univ, CNRS, LAM, Laboratoire d'Astrophysique de Marseille, Marseille, France

${ }^{14}$ CRAL, UMR 5574, CNRS, Université de Lyon, Ecole Normale Supérieure de Lyon, 46 Allée d'Italie, 69364 Lyon Cedex 07, France

15 INAF-Osservatorio Astronomico di Brera, Via E. Bianchi 46, 23807 Merate, Italy

16 INCT-Universidad De Atacama, calle Copayapu 485, Copiapó Atacama, Chile
17 Department of Astronomy, University of Michigan, 1085 S. University Ave, Ann Arbor, MI 48109-1107, USA

18 Leiden Observatory, Leiden University, PO Box 9513, 2300 RA Leiden, The Netherlands

19 Physikalisches Institut, Universität Bern, Gesellschaftsstrasse 6, 3012 Bern, Switzerland

20 Konkoly Observatory Research, Centre for Astronomy and Earth Sciences, Hungarian Academy of Sciences, PO Box 67, 1525 Budapest, Hungary

21 Aix Marseille Univ, CNRS, LAM, Laboratoire d'Astrophysique de Marseille, UMR 7326, 13388 Marseille, France

22 European Southern Observatory (ESO), Alonso de Còrdova 3107, Vitacura, Casilla 19001, Santiago, Chile

23 Núcleo de Astronomía, Facultad de Ingeniería y Ciencias, Universidad Diego Portales, Av. Ejercito 441, Santiago, Chile

24 Escuela de Ingeniería Industrial, Facultad de Ingeniería y Ciencias, Universidad Diego Portales, Av. Ejercito 441, Santiago, Chile 


\section{Appendix A: Determination of host star properties}

We used a Markov chain Monte Carlo approach to find the posterior distribution for the PDS 70 host star parameters, adopting the emcee code (Foreman-Mackey et al. 2013). The unknown parameters are the stellar mass, age, extinction, and parallax ${ }^{1}$, and we assumed solar metallicity. The photometric measurements used for the fit, and the independently determined effective temperature $T_{\text {eff }}$ and radius are listed in Table A.1. We perform a simultaneous fit of all these observables. The uncertainties are treated as Gaussians and we assume no covariance between them.

We used a Gaussian prior from Gaia for the distance and a Gaussian prior with mean $0.01 \mathrm{mag}$ and sigma $0.07 \mathrm{mag}$, truncated at $A_{\mathrm{V}}=0 \mathrm{mag}$, for the extinction (Pecaut \& Mamajek 2016). Given $A_{\mathrm{V}}$, we computed the extinction in all the adopted bands by assuming a Cardelli et al. (1989) extinction law. We used a Chabrier (2003) initial mass function (IMF) prior on the mass and a uniform prior on the age. The stellar models adopted to compute the expected observables, given the fit parameters, are from the MIST project (Paxton et al. 2011, 2013, 2015; Dotter 2016; Choi et al. 2016). These models were extensively tested against young cluster data, and against pre-main sequence stars in multiple systems, with measured dynamical masses, and compared to other stellar evolutionary models (see Choi et al. 2016 for details). The result of the fit constrains the age of PDS 70 to $5.4 \pm 1.0 \mathrm{Myr}$ and its mass to $0.76 \pm 0.02 M_{\odot}$. The best-fit parameter values are given by the $50 \%$ quantile (the median) and their uncertainties are based on the $16 \%$ and $84 \%$ quantile of the marginalized posterior probability distribution. The stellar parameters are identical to the values used by Keppler et al. (2018). We note that the derived stellar age of PDS 70 is significantly younger than the median age derived for UCL with $16 \pm 2 \mathrm{Myr}$ and an age spread of 7 Myr by Pecaut \& Mamajek (2016). For the computation of the median age Pecaut \& Mamajek (2016) excluded K- and M-type stars for the reason of stellar activity which might bias the derived age. When the entire sample of stars is considered a median age of $9 \pm 1 \mathrm{Myr}$ is derived. The authors provide an age of $8 \mathrm{Myr}$ for PDS 70 based on evolutionary models. Furthermore, the kinematic parallax for PDS 70 therein is larger by $\sim 15 \%$ compared to the new Gaia parallax. Thus, the luminosity on which the age determination is based is underestimated and, subsequently, the age is overestimated.

\section{Appendix B: Disk seen with IRDIS}

Figure B. 1 shows the IRDIS combined $K_{1} K_{2}$ image using classical ADI. The image shows the outer disk ring, with a radius of approximately $54 \mathrm{au}$, with the western (near) side being brighter than the eastern (far) side, as in Hashimoto et al. (2012) and Keppler et al. (2018). The image reveals a highly structured disk with several features: 1) a double ring structure along the west side, which is clearly pronounced along the northwest arc, and which is less clear but still visible along the southwest side; 2) a possible connection from the outer disk to the central region; $3,4)$ a possible spiral-shaped feature close to the coronagraph; and 5) two arc-like features in the gap on the southeast side of

\footnotetext{
1 The parallax of PDS 70 is treated as an unknown parameter in our fit to the host star's properties, together with mass, age and $A_{\mathrm{V}}$. However we imposed a parallax prior, using Gaia DR2, which strongly constrains the allowed distance values. As a result, the best-fit distance value reported here from the MCMC posterior draws is identical to the value provided by the Gaia collaboration.
}

Table A.1. Stellar parameters of PDS 70.

\begin{tabular}{lccc}
\hline \hline Parameter & Unit & Value & References \\
\hline Distance & $\mathrm{pc}$ & $113.43 \pm 0.52$ & 1 \\
$T_{\text {eff }}$ & $\mathrm{K}$ & $3972 \pm 36$ & 2 \\
Radius & $R_{\odot}$ & $1.26 \pm 0.15$ & Computed from 2 \\
$B$ & $\mathrm{mag}$ & $13.494 \pm 0.146$ & 3 \\
$V$ & $\mathrm{mag}$ & $12.233 \pm 0.123$ & 3 \\
$g^{\prime}$ & $\mathrm{mag}$ & $12.881 \pm 0.136$ & 3 \\
$r^{\prime}$ & $\mathrm{mag}$ & $11.696 \pm 0.106$ & 3 \\
$i^{\prime}$ & $\mathrm{mag}$ & $11.129 \pm 0.079$ & 3 \\
$J$ & $\mathrm{mag}$ & $9.553 \pm 0.024$ & 4 \\
$H$ & $\mathrm{mag}$ & $8.823 \pm 0.040$ & 4 \\
$K_{\mathrm{s}}$ & $\mathrm{mag}$ & $8.542 \pm 0.023$ & 4 \\
Age & $\mathrm{Myr}$ & $5.4 \pm 1.0$ & This work \\
Mass & $M_{\odot}$ & $0.76 \pm 0.02$ & This work \\
$A_{\mathrm{V}}$ & $\mathrm{mag}$ & $0.05_{-0.03}^{+0.05}$ & This work \\
\hline
\end{tabular}

References.(1) Gaia Collaboration (2016, 2018); (2) Pecaut \& Mamajek (2016); (3) Henden et al. (2015); (4) Cutri et al. (2003).

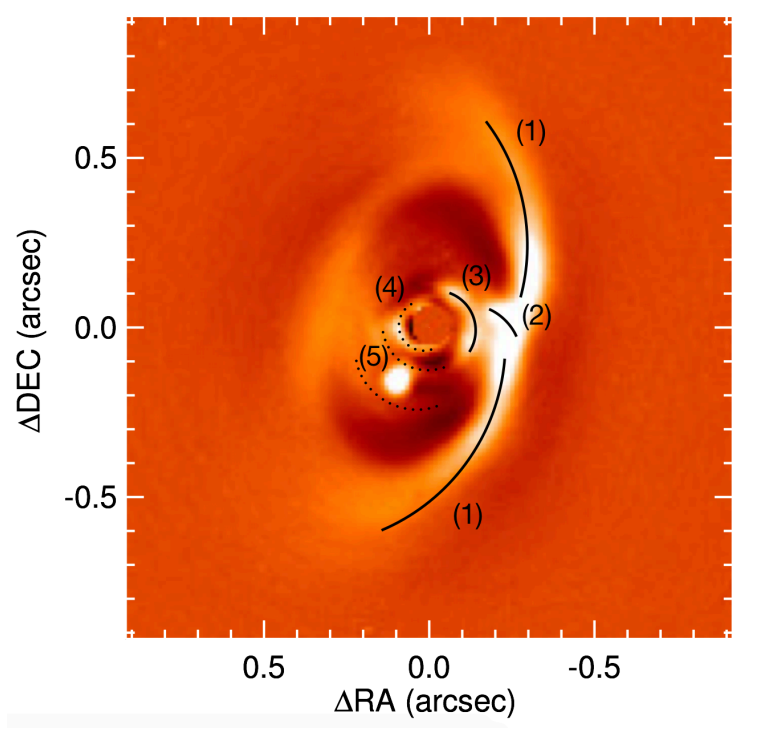

Fig. B.1. IRDIS combined $K_{1} K_{2}$ image of PDS 70 using classical ADI. To increase the dynamic range of the faint disk structures, the companion's full intensity range is not shown. The black lines indicate the structures discussed in the above text. North is up, east is to the left.

the central region. Whereas features 1 and 2 have already been tentatively seen in previous observations (see Figs. 5 and 9 in Keppler et al. 2018), our new and unprecedentedly deep data set allows us to identify extended structures well within the gap (features 3-5). Future observations at high resolution, i.e., with interferometry, will be needed to prove the existence and to investigate the nature of these features, which, if real, would provide an excellent laboratory for probing theoretical predictions of planetdisk interactions. 


\section{Appendix C: Astrometric and photometric detailed}

\section{results}

Table C.1. Relative astrometry and photometry of PDS $70 \mathrm{~b}$ as derived from the sPCA reduction.

\begin{tabular}{cccccccccc}
\hline \hline Date & Instr. & Filter & $\Delta \alpha$ (mas) & $\Delta \delta$ (mas) & Sep. (mas) & PA (deg) & $\Delta$ mag & mag $_{\text {app }}$ & Peak $S / N$ \\
\hline $2012-03-31$ & NICI & $L^{\prime}$ & $58.7 \pm 10.7$ & $-182.7 \pm 22.2$ & $191.9 \pm 21.4$ & $162.2 \pm 3.7$ & $6.59 \pm 0.42$ & $14.50 \pm 0.42$ & 5.6 \\
$2015-05-03$ & IRDIS & $H 2$ & $83.1 \pm 3.9$ & $-173.5 \pm 4.3$ & $192.3 \pm 4.2$ & $154.5 \pm 1.2$ & $9.35 \pm 0.18$ & $18.17 \pm 0.18$ & 6.3 \\
$2015-05-03$ & IRDIS & $H 3$ & $83.9 \pm 3.6$ & $-178.5 \pm 4.0$ & $197.2 \pm 4.0$ & $154.9 \pm 1.1$ & $9.24 \pm 0.17$ & $18.06 \pm 0.17$ & 8.1 \\
$2015-05-31$ & IRDIS & $H 2$ & $89.4 \pm 6.0$ & $-178.3 \pm 7.1$ & $199.5 \pm 6.9$ & $153.4 \pm 1.8$ & $9.12 \pm 0.24$ & $17.94 \pm 0.24$ & 11.4 \\
$2015-05-31$ & IRDIS & $H 3$ & $86.9 \pm 6.2$ & $-174.0 \pm 6.4$ & $194.5 \pm 6.3$ & $153.5 \pm 1.8$ & $9.13 \pm 0.16$ & $17.95 \pm 0.17$ & 6.8 \\
$2016-05-14$ & IRDIS & $K 1$ & $90.2 \pm 7.3$ & $-170.8 \pm 8.6$ & $193.2 \pm 8.3$ & $152.2 \pm 2.3$ & $7.81 \pm 0.31$ & $16.35 \pm 0.31$ & 5.5 \\
$2016-05-14$ & IRDIS & $K 2$ & $95.2 \pm 4.8$ & $-175.0 \pm 7.7$ & $199.2 \pm 7.1$ & $151.5 \pm 1.6$ & $7.67 \pm 0.24$ & $16.21 \pm 0.24$ & 3.6 \\
$2016-06-01$ & NaCo & $L^{\prime}$ & $94.5 \pm 22.0$ & $-164.4 \pm 27.6$ & $189.6 \pm 26.3$ & $150.6 \pm 7.1$ & $6.84 \pm 0.62$ & $14.75 \pm 0.62$ & 2.7 \\
$2018-02-24$ & IRDIS & $K 1$ & $109.6 \pm 7.9$ & $-157.7 \pm 7.9$ & $192.1 \pm 7.9$ & $147.0 \pm 2.4$ & $8.10 \pm 0.05$ & $16.65 \pm 0.06$ & 16.3 \\
$2018-02-24$ & IRDIS & $K 2$ & $110.0 \pm 7.9$ & $-157.6 \pm 8.0$ & $192.2 \pm 8.0$ & $146.8 \pm 2.4$ & $7.90 \pm 0.05$ & $16.44 \pm 0.05$ & 13.7 \\
\hline
\end{tabular}

Notes. For completeness we list the values from the first five epochs from Keppler et al. (2018). The astrometric values are corrected for true north, and account for the instrument anamorphism (Maire et al. 2016). The true north correction for the IRDIS data recorded on February 24, 2018, is $-1.76^{\circ} \pm 0.06^{\circ}$. For true north values from earlier epochs, see Table 4 in Keppler et al. (2018).

\section{Appendix D: Markov chain Monte Carlo results}

Table D.1. MCMC solutions for the orbital parameters of PDS $70 \mathrm{~b}$.

\begin{tabular}{ccccccccccc}
\hline \hline & & \multicolumn{4}{c}{ Unrestrained solutions } & & \multicolumn{3}{c}{ Solutions for restrained $i$ and $\Omega$} \\
\cline { 3 - 5 } Parameter & Unit & Peak & Median & Lower & Upper & & Peak & Median & Lower & Upper \\
\hline \multirow{2}{*}{$a$} & au & 22.2 & 25.1 & 12.5 & 28.4 & & 21.2 & 23.8 & 13.3 & 27.0 \\
$e$ & & 0.03 & 0.17 & 0.0 & 0.25 & & 0.03 & 0.18 & 0.0 & 0.27 \\
$i$ & $\circ$ & 151.1 & 150.1 & 137.5 & 165.2 & & 131.1 & 131.0 & 128.3 & 133.6 \\
$\Omega$ & $\circ$ & -128.1 & 0.0 & -180.0 & 51.0 & & 159.6 & 158.4 & 156.2 & 163.9 \\
$\omega$ & $\circ$ & -130.9 & 0.0 & -180.0 & 59.9 & & -12.7 & 2.5 & -144.7 & 52.3 \\
$t_{\mathrm{p}}$ & $\mathrm{yr}$ & 2041.9 & 2020.1 & 2001.4 & 2069.0 & 2009.1 & 2013.4 & 1973.1 & 2029.1 \\
\hline
\end{tabular}

Notes. The left part of the table lists the values obtained without any prior information taken into account. The right part of the table lists the solution for the restrained case. The lower and upper values correspond to the $68 \%$ confidence interval. 
A. Müller et al.: Orbital and atmospheric characterization of the planet within the gap of the PDS 70 transition disk

$\begin{array}{ccccc}a(\mathrm{AU}) & \mathrm{e} \\ 20 & 40 \quad 60 \quad 80 & 1000.2 & 0.4 & 0.6\end{array}$ $\begin{array}{llllll}20 & 40 \quad 60 \quad 80 & 1000.2 & 0.4 & 0.6 & 0.8\end{array}$ $\mathrm{i}\left({ }^{\circ}\right)$ $\omega\left(^{\circ}\right)$ $t_{p}$

0200020502100

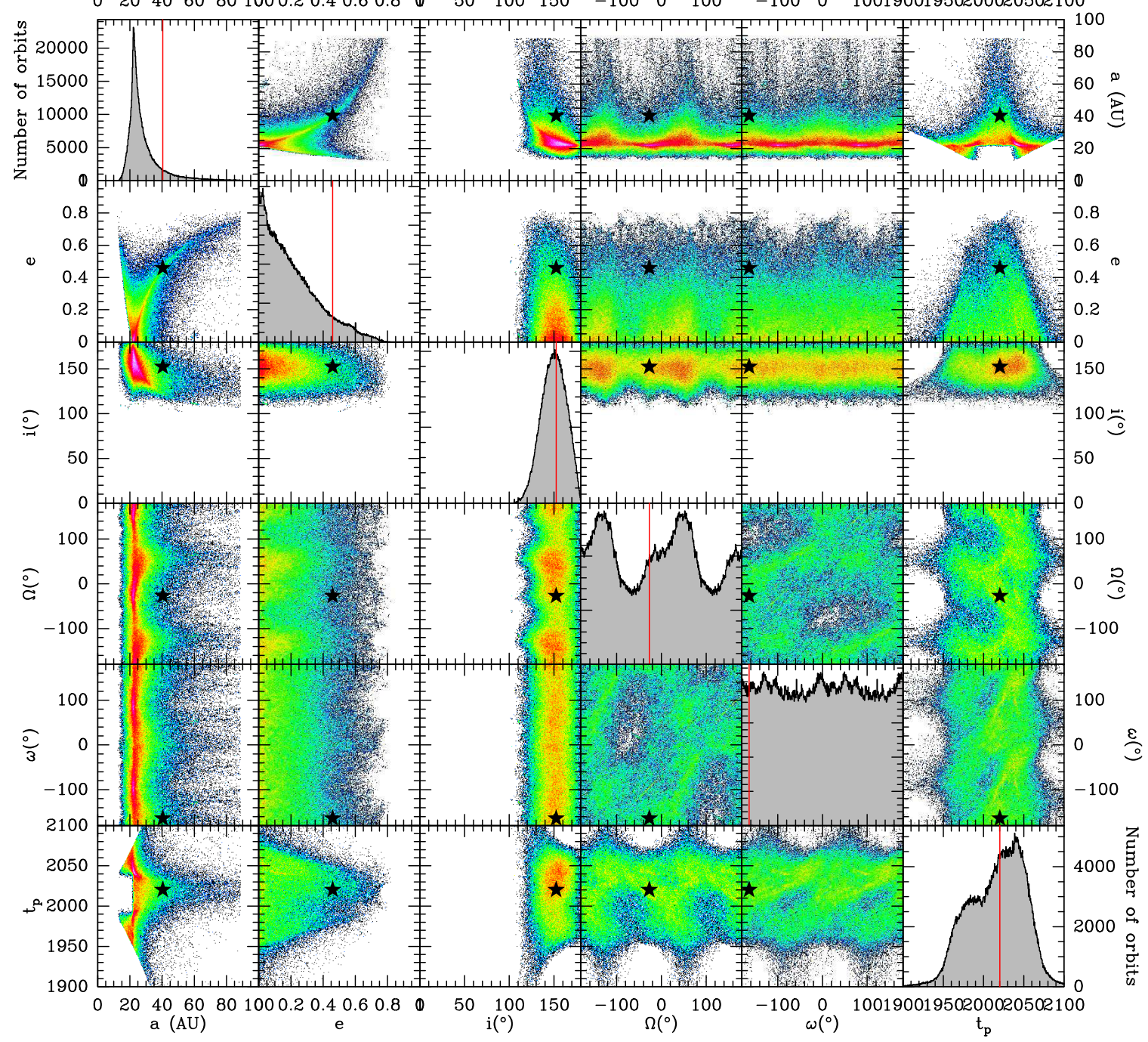

Fig. D.1. Results of the MCMC fit of the SPHERE, NaCo, and NICI combined astrometric data of PDS $70 \mathrm{~b}$ reported in terms of statistical distribution matrix of the orbital elements $a, e, i, \Omega, \omega$, and $t_{\mathrm{p}}$. The red line in the histograms and the black star in the correlation plots indicate the position of the best LSLM $\chi_{\mathrm{r}}^{2}$ model obtained for comparison. 
a $(\mathrm{AU})$

$\mathrm{i}\left({ }^{\circ}\right)$

$\Omega\left({ }^{\circ}\right)$

$\omega\left(^{\circ}\right)$

$t_{p}$

$0204060801000.20 .40 .60 .8120125130135146150155160165170-100 \quad 0 \quad 10019001950200020502100$

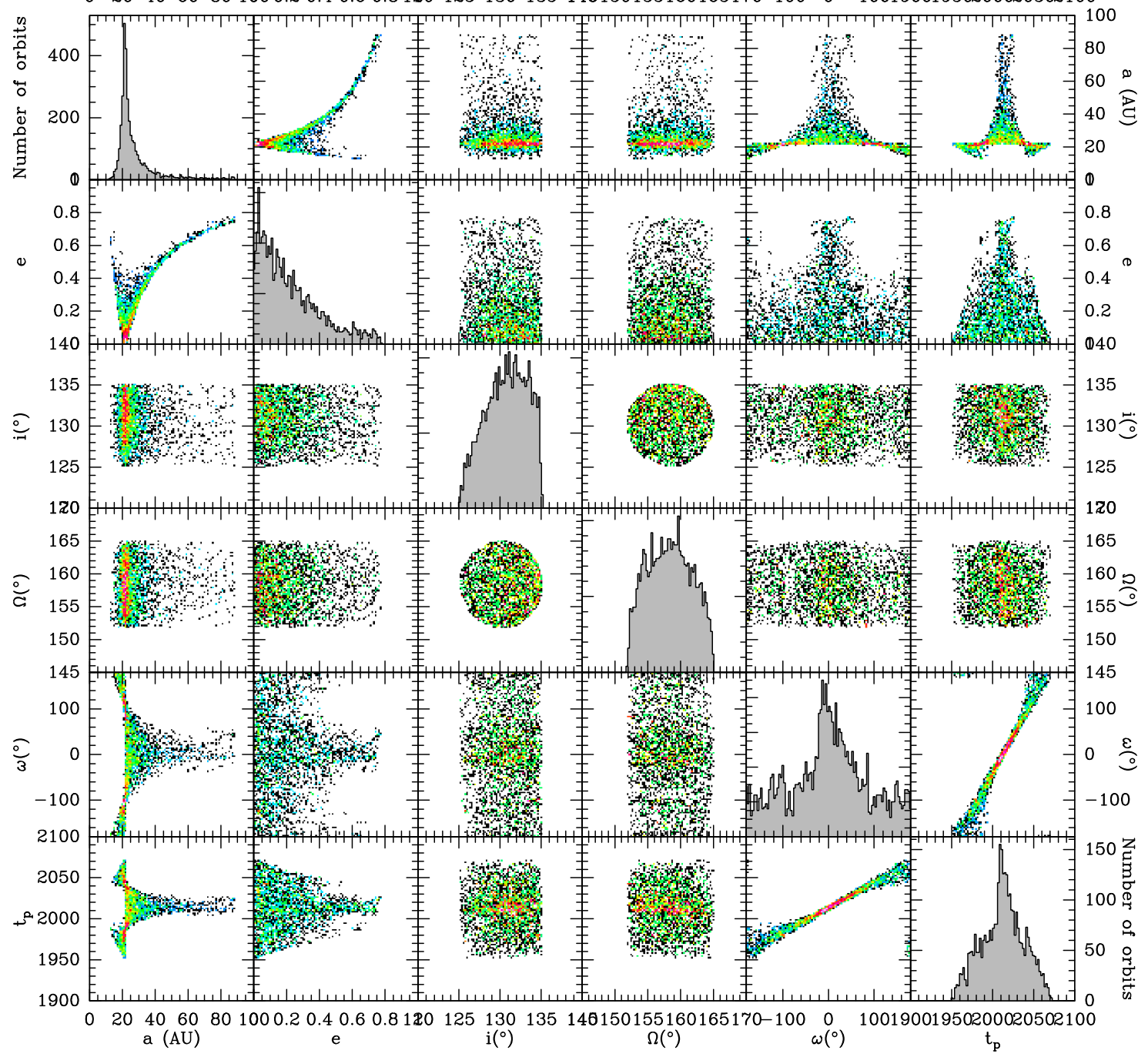

Fig. D.2. Results of the MCMC fit of the SPHERE, NaCo, and NICI combined astrometric data of PDS $70 \mathrm{~b}$ reported in terms of statistical distribution matrix of the orbital elements $a, e, i, \Omega, \omega$, and $t_{\mathrm{p}}$. We restrained the PDS70 b solution set given by the MCMC to solutions with orbital plane making a tilt angle of less than $5^{\circ}$ with respect to the disk midplane described by Keppler et al. (2018), i.e., $i=180^{\circ}-49.8^{\circ}$ and PA $=158.6^{\circ}$.

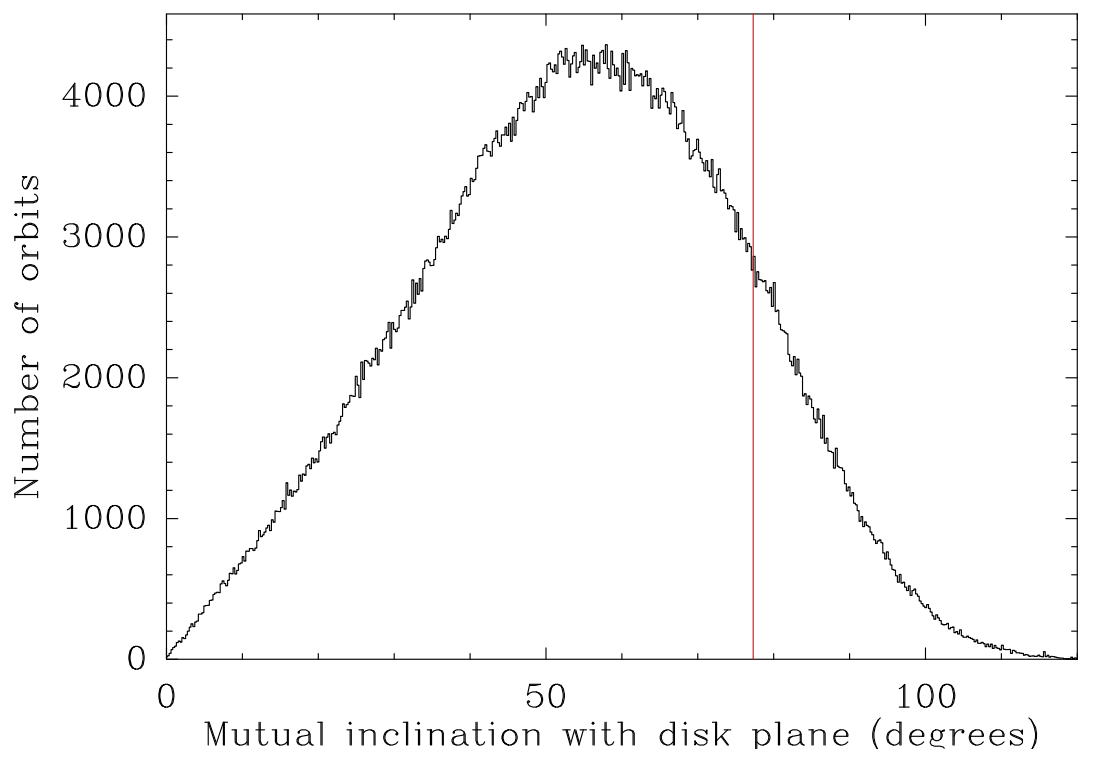

Fig. D.3. Posterior distribution (from Fig. D.1) of the tilt angle. The distribution peaks around $50^{\circ}$, which remains consistent with a likely coplanar planet-disk configuration. The red line indicates the position of the best LSLM $\chi_{\mathrm{r}}^{2}$ model obtained for comparison. 
A. Müller et al.: Orbital and atmospheric characterization of the planet within the gap of the PDS 70 transition disk

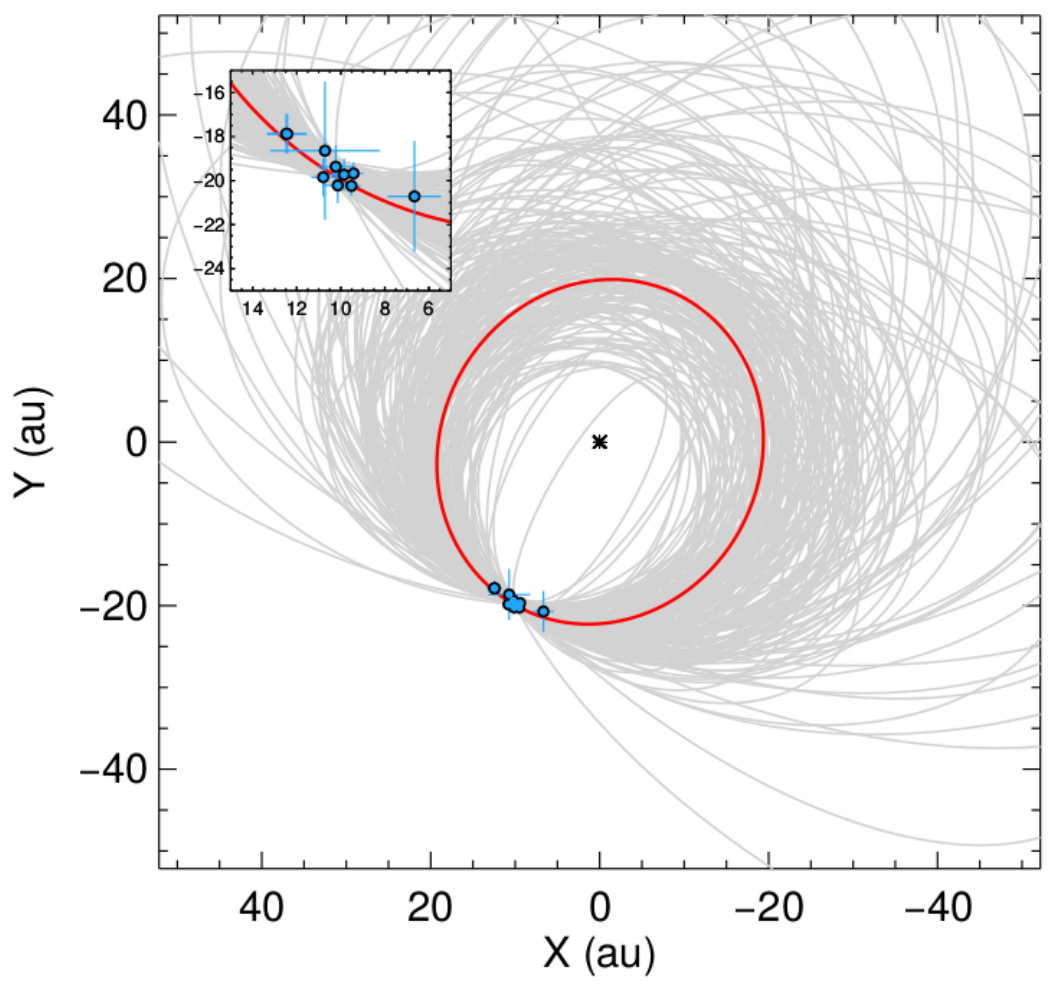

Fig. D.4. Relative astrometry of PDS $70 \mathrm{~b}$ solutions drawn from the MCMC distribution for the coplanar planet-disk configuration. One of the most likely solutions from our MCMC analysis is shown as an illustration (in red). 